\title{
AN INVISCID THEORY FOR SLIDING FLEXIBLE SHEETS
}

\author{
E. O. TUCK
}

(Received 28 August 1980)

(Revised 30 May 1981)

\begin{abstract}
This paper demonstrates feasibility of aerodynamically-supported motion of a thin sheet near to a plane wall. Steady equilibrium is possible for uniform sheets only if they are deformable, and a set of possible equilibrium shapes is determined.
\end{abstract}

\section{Introduction}

The phenomenon of easy sliding of a light flat sheet over a plane surface is familiar to all. A vivid demonstration is contained in the movie prepared by the late Sir Geoffrey Taylor [4]. Taylor noted that impermeability of the sheet is important, and demonstrated that a sheet with holes cannot slide. He included this demonstration in a film that is otherwise devoted to low-Reynolds-number fluid mechanics, and described the sliding phenomenon as an example of fluid lubrication, in which viscosity is the most important fluid property.

Our purpose here is to analyse the alternative possibility that support for the weight of a sliding sheet can come from conventional aerodynamic lift due to circulation, even in the absence of viscosity. Any such tendency for lift development is strongly augmented by ground effect, in situations like the present where the distance from the ground is small compared to the horizontal dimensions of the lifting surface.

The aerodynamics of lifting surfaces very close to the ground can be analysed by a quite simple one-dimensional theory, perhaps first due to Strand, Royce and Fujita [3], that was extended by Widnall and Barrows. [7] and, more recently, by

CCCopyright Australian Mathematical Society 1982 
the present author [5], [6]. This theory (in its steady non-linear form, [6]) predicts a pressure distribution in the gap that varies as the negative inverse square of the local gap width, and takes values comparable in magnitude over a large portion of the sheat to the maximum possible (stagnation) pressure in an inviscid fluid.

Thus, a sufficiently light sheet can be supported aerodynamically. However there is a difficulty for uniform rigid sheets in that the centre of aerodynamic pressure is not in general located at the centre of the sheet. Hence, such a sheet cannot be in equilibrium. The escape from this difficulty is to assume that the sheet is flexible and responds to a distributed aerodynamic pressure by bending slightly.

In the present work, we are able to solve such coupled aerodynamic-bending problems for the equilibrium configurations. The solution is not unique, there being in principle infinitely many shapes that the sheet can deform into, such that the lift exactly supports the sheet's weight, and acts at its centre. The lowest-order mode is symmetric about the centre, where its clearance is greatest. This mode also has a much higher average clearance than all other modes, and would presumably be the most likely mode for successful sliding, since its drag would be less.

\section{Problem formulation}

The flow of interest is as sketched in Figure 1. Viewed in a frame of reference fixed in the sheet, the flow is steady and two-dimensional, and has a uniform stream $U$ in the negative direction at infinity. The ground surface is the plane $y=0$, and the sheet is fixed at the position $y=y(x)$. The fluid is assumed inviscid and incompressible. The sheet is assumed to be of zero thickness (for aerodynamic purposes) and to lie between $x=0$ (trailing edge) and $x=L$ (leading edge), so having chord $L$. The clearance $y(x)$ is assumed everywhere small compared to $L$. This means that the slope or local angle of attack $y^{\prime}(x)$ is everywhere small.

The sheet is also assumed to be flexible, allowing bending in response to the aerodynamic pressures. Thus, we have to solve a coupled aerodynamic-elastic problem in which the aerodynamic pressure is determined by the bending shape function $y(x)$ which in turn is determined by the aerodynamic pressure.

The aerodynamic portion of this problem is a well-posed potential-flow problem, which could be attacked by the usual methods of theoretical aerodynamics. Circulation is induced around the sheet by the requirement (the Kutta condition) that the flow streamlines leave the trailing edge $x=0$ of the sheet smoothly, and this circulation is ultimately responsible for the lift that supports the sheet's weight. 


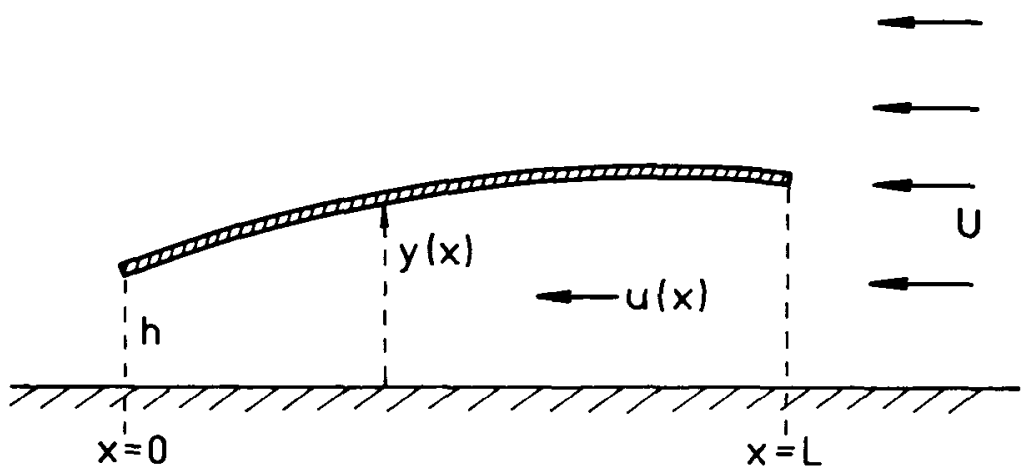

Figure 1. Sketch of fixed sheet $y=y(x)$ in a steady stream $U$.

However, the predominant feature of the present configuration is the presence of the ground plane, which augments the lift by (in effect) contributions due to images in that ground plane, of the apparent vortices above it. In the limit as $y / L \rightarrow 0$, that augmentation becomes so great that a special asymptotic theory applies, and predicts lift forces orders of magnitude greater than those for a lifting surface without ground effect. This theory will now be described.

\section{Aerodynamic detail}

If $u(x)$ is the velocity of the predominantly one-dimensional flow in the narrow. gap, between the plane $y=0$ and the sheet $y=y(x)$, then continuity demands that $u(x) y(x)$ is constant, and equal to its value at a specified value of $x$. We choose as that specified position the trailing edge $x=0$, writing $h=y(0)$ for the trailing-edge clearance.

In addition, we make use of the Kutta, or smooth-detachment, condition at the trailing edge, to assert that the gap speed $u(0)$ at that point is equal to the free-stream speed $U$. This is an important assumption, of course, and applies only to trailing edges that are locally very straight, that is, free from bends or flaps. Matching procedures described in [5] and [6] can be used to justify this assumption in the asymptotic limit as the clearance/chord ratio vanishes. In particular, [6], a correction can be made when there do exist local bends or flaps near the trailing edge, but we shall assume here that no such deviations occur.

Thus we have

$$
u(x) y(x)=U h
$$


Now, if $p$ denotes the excess of pressure over the free-stream value, then Bernoulli's equation states that, for an inviscid fluid of constant density $\rho$ in steady flow,

$$
p / \rho+\frac{1}{2} q^{2}=\frac{1}{2} U^{2}
$$

where $q$ is the fluid velocity magnitude. In the gap, $q \simeq u(x)$, because of the predominantly one-dimensional character of the flow, and hence

$$
p=\frac{1}{2} \rho U^{2}-\frac{1}{2} \rho u^{2}=\frac{1}{2} \rho U^{2}\left(1-h^{2} / y^{2}\right),
$$

upon use of the continuity equation (3.1).

Equation (3.3) is the fundamental aerodynamic result for steady ground-effect flows. It shows that the gap pressure is an $O(1)$ multiple $\left(1-h^{2} / y^{2}\right)$ of the stagnation pressure $\frac{1}{2} \rho U^{2}$. The net lift is

$$
F=\frac{1}{2} \rho U^{2} \int_{0}^{L}\left[1-h^{2} / y^{2}(x)\right] d x
$$

and the moment (leading edge up) about mid-chord is

$$
M=\frac{1}{2} \rho U^{2} \int_{0}^{L}\left[1-h^{2} / y^{2}(x)\right](x-L / 2) d x .
$$

These results assume that pressure variations along the upper surface of the sheet are negligible compared to the gap pressure.

An important special case is that of a flat plate at angle of attack $\alpha$, where

$$
y(x)=h+\alpha x \text {. }
$$

The integrals in (3.4) and (3.5) can be evaluated easily; in particular, we find the net lift

$$
F=\frac{1}{2} \rho U^{2} L \alpha /(\alpha+h / L)
$$

Note that for small angle of attack $\alpha$, the lift is proportional to $\alpha$, as is usual in thin airfoil theory. However, "small" in the present context means small sompared to $h / L$, which is itself a very small quantity. More realistically, $\alpha$ is expected to be comparable to $h / L$, and then (3.7) shows that the lift coefficient is the ratio

$$
\frac{\alpha L}{h+\alpha L}=\frac{\text { Leading edge clearance-Trailing edge clearance }}{\text { Leading edge clearance }},
$$

which takes its maximum value of unity when $h=0$ (that is, trailing edge touches ground), and tends to negative infinity as $h+\alpha L \rightarrow 0$ (that is, leading edge touches ground).

The corresponding expression for the moment $M$ of a rigid flat plate at angle of attack $\alpha$ is given in [6], together with a discussion of the static stability problem. All we need note about that result at the present time is that $M=0$ only if $h=0$. That is, unless the gap flow is completely stalled (and hence the pressure is 
uniform) the centre of pressure cannot lie at mid-chord. For positive lift $(\alpha>0)$, the centre of pressure is forward of mid-chord, approaching the $\frac{1}{3}$-chord point as $\alpha \rightarrow 0$.

It is therefore apparent that a uniform rigid sheet cannot slide steadily (with non-zero clearance everywhere) over a plane surface, with lift being provided by aerodynamic forces alone. If the weight of the sheet is uniformly distributed, its centre of gravity will be at mid-chord, and even if enough aerodynamic lift is available to support that weight, there will still be a net moment tending to increase the angle of attack. Of course, such a tendency will lead to a reduction in the trailing-edge clearance $h$, and hence, ultimately, to a stalled gap flow. This represents (in one sense) a desirable situation of maximum lift, acting at mid-chord. Meanwhile, viscous effects would have played a significant role in the locally restricted flow near the trailing edge, and, in particular, we must expect a significant increase in drag.

The only alternative is that the sheet is allowed to deform under the action of aerodynamic forces, and we now assume that this is possible. The deformations must be such that the net moment $M$ given by (3.5) is zero, so that the lift acts at the mid-chord position of the centre of gravity. Certainly, there do exist bending modes allowing a net positive lift, and having that property. In particular, all modes that are symmetric about mid-chord necessarily have $M=0$, and the lift is positive if (for example) $y(x)>h$ for all $x \neq 0$ and $L$. However, this is not the only possibility; in particular, as we shall see, a nearly-rigid plate at an angle of attack can be made to shift its centre of pressure to mid-chord, by distorting it in a roughly anti-symmetric manner, to give greater clearance in the aft half of the sheet.

We now analyze the bending of deformable sheets in detail.

\section{Bending modes}

The sheet is now assumed to be a flexible thin shell, with flexural rigidity

$$
D=\frac{E t^{3}}{12\left(1-\sigma^{2}\right)},
$$

where $E$ is Young's modulus, $t$ the sheet's thickness, and $\sigma$ Poisson's ratio. In steady equilibrium with shape $y=y(x)$, the equations of thin shell theory (Donnell [2]) state that

$$
\frac{d^{2}}{d x^{2}}\left[D \frac{d^{2} y}{d x^{2}}\right]=p(x)-w
$$


where $w$ is the sheet's weight per unit area, and $p(x)$ the net (upward) force per unit area due to fluid effects. We assume that the sheet is uniform in all properties, that is, that $D$ and $w$ are constant, and that $p(x)$ can be equated with the gap pressure given by equation (3.3).

Thus, the equation of equilibrium is

$$
D y^{\text {iv }}=\left(\frac{1}{2} \rho U^{2}-w\right)-\left(\frac{1}{2} \rho U^{2} h^{2}\right) y^{-2} .
$$

This is a non-linear ordinary differential equation of the 4th order. It must be solved subject to the 4 free-end conditions

$$
y^{\prime \prime}=y^{\prime \prime \prime}=0 \text { at } x=0 \text { and } L,
$$

and an apparent 5 th boundary condition, namely

$$
y(0)=h .
$$

However, $h$ is itself an unknown; hence the combined system (4.3)-(4.5) is properly set as a 4 th-order boundary-value problem, with $h$ to be determined as part of that problem.

It is immediately clear that no solution exists if $w>\frac{1}{2} \rho U^{2}$. Mathematically, this means that the expression on the right of (4.3) is everywhere negative; if $y^{\text {iv }}<0, y^{\prime \prime \prime}$ cannot vanish at both $x=0$ and $x=L$. Physically, of course, $\frac{1}{2} \rho U^{2}$ is the maximum attainable aerodynamic pressure, and aerodynamics alone cannot support the weight of a sheet with $w>\frac{1}{2} \rho U^{2}$.

At the other extreme, if $w$ is very small compared to $\frac{1}{2} \rho U^{2}$, aerodynamic forces are ample to support the weight, and little bending is needed. This corresponds to the linear ground-effect theory of Widnall and Barrows [7]. Thus, when $w \ll$ $\frac{1}{2} \rho U^{2}, y \simeq h$ and the non-linear equation (4.3) can be linearized $\left(y^{-2} \simeq h^{-2}-\right.$ $\left.2 h^{-3}(y-h)\right)$ to give

$$
D y^{\text {iv }}-\left(\rho U^{2} / h\right) y=-w-\rho U^{2} .
$$

The general solution of (4.6) can be written down at once in terms of trigonometric and hyperbolic functions of $k x$, where

$$
k^{4}=\rho U^{2} /(D h) \text {. }
$$

In particular,

$$
y-h=\left(h w /\left(\rho U^{2}\right)\right)\left[1-\frac{1}{2}(\cos k x+\cosh k x)+\beta(\sin k x+\sinh k x)\right] \text {, }
$$

satisfies $y=h$ and $y^{\prime \prime}=y^{\prime \prime \prime}=0$ at $x=0$, for any constant $\beta$. The condition $y^{\prime \prime}=y^{\prime \prime \prime}=0$ at $x=L$ is satisfied if

$$
\cos k L=\operatorname{sech} k L,
$$


a transcendental equation from which an infinite set of eigenvalues for $k L$ may be obtained, namely

$$
k L=4.730 . ., 7.853 . ., 10.996 . ., . .=K_{1}, K_{2}, K_{3}, \ldots
$$

In fact, beyond the 2 nd, these eigenvalues are to 3 decimal places indistinguishable from odd multiples of $\pi / 2$, since sech $k L \simeq 0$.

For each eigenvalue $k L=K_{n}, n=0,1,2, \ldots$, above, the corresponding value of $\beta=\beta_{n}$ may be determined. In fact, the solution (4.8) may then be re-written in such a way as to demonstrate a symmetry property that is in any case evident from the nature of the linearized problem. That is, the even eigenvalues $n=0,1, \ldots$ generate solutions that are symmetric about the mid-chord position $x=L / 2$, with $y(L / 2-x)=y(L / 2+x)$ while the odd eigenvalues generate exactly antisymmetric modes, with $y^{\prime}(L / 2-x)=y^{\prime}(L / 2+x)$.

The eigenvalues for $k L$ generate corresponding eigenvalues for the trailing-edge clearance $h$, that is,

$$
h=\rho U^{2} /\left(D k^{4}\right)=\left(\rho U^{2} L^{4} / D\right) K_{n}^{-4} .
$$

Note that, as $n \rightarrow \infty, K_{n} \rightarrow \infty$ and $h \rightarrow 0$ very rapidly, because of the inverse 4th power. That is, as is to be expected physically, complex modes of bending, with many maxima and minima, are less efficient in generating lift, and hence the whole sheet must be closer to the ground to support its weight. Such modes are therefore more influenced by viscous effects and in particular will suffer a greater drag penalty.

We now turn to the fully non-linear problem, specified by equation (4.3). It is interesting to note that the symmetric linearized solutions for $n=0,2,4, \ldots$ will generalize to (exactly) symmetric non-linear solutions. That is, the non-linear problem possesses solutions satisfying $y(L / 2-x)=y(L / 2+x)$. However, the non-linear problem is not invariant under the transformation $y^{\prime}(L / 2-x)=$ $y^{\prime}(L / 2+x)$, and hence we cannot expect any (exactly) antisymmetric modes, although there must be modes which become anti-symmetric in the limit as $w \rightarrow 0$.

\section{Numerical method and computed solutions}

The non-linear boundary-value problem (4.3)-(4.5) can be converted, in part, to an initial-value problem as follows. First perform a scaling, writing

$$
y(x)=h Y(k x)
$$

where $k$ is as defined in (4.7). Then $Y=Y(X)$ satisfies

$$
2 \frac{d^{4} Y}{d X^{4}}=1-W-Y^{-2}
$$


where

$$
W=w /\left(\frac{1}{2} \rho U^{2}\right) .
$$

Equation (5.2) is to be solved for fixed $W$ where $0<W<1$, subject to

$$
Y=1, Y^{\prime \prime}=Y^{\prime \prime \prime}=0 \text { at } X=0,
$$

and

$$
Y^{\prime \prime}=Y^{\prime \prime \prime}=0 \text { at } X=k L .
$$

For numerical purposes, we augment the set of conditions (5.4) at $X=0$ by the slope condition

$$
Y^{\prime}(0)=\gamma,
$$

where $\gamma$ is an unknown constant. The initial-value problem corresponding to (5.2) subject to (5.4), (5.6), has a unique solution for any $\gamma$. Such a solution can be obtained easily by various algorithms, such as the Runge-Kutta method. Our task is thus to solve a family of such initial-value problems for various $\gamma$, and to seek a particular value of $\gamma$ such that (5.5) is satisfied at some value of $X=K_{n}>0$.

For the even modes $n=0,2, \ldots$, this computational task can be reduced by using the symmetry property. That is, instead of continuing the computation to $X=K_{n}$ we can stop at a pont $X=\frac{1}{2} K_{n}$ where $Y^{\prime}=Y^{\prime \prime \prime}=0$. However, to generate the remaining (nearly-antisymmetric) modes $n=1,3, \ldots$, we must compute over a large range, until (5.5) is satisfied.

In practice, it is only necessary to examine the zeros of the second derivative $Y^{\prime \prime}$, as $\gamma$ varies. Rolle's theorem guarantees that there is a zero of $Y^{\prime \prime \prime}$ between any two successive zeros of $Y^{\prime \prime}$, so that any point where $Y^{\prime \prime}$ and $Y^{\prime \prime \prime}$ vanish simultaneously must be a point where two zeros of $Y^{\prime \prime}$ coalesce. Hence, if we plot zeros of $Y^{\prime \prime}$ against $\gamma$, the required solutions correspond to values of $\gamma$ where that plot is vertical.

A typical such plot is shown as Figure 2, for $W=0.5$. Note that the relevant values of $\gamma$ lie in a very narrow range, in which many turning points appear to occur. Accuracy is ultimately lost as $X$ increases, so that it is not possible to say from the numerical values that an infinite number of turning points (and hence an infinite number of eigenvalues) exist, but it seems likely that this is so. In any case, we are only really interested in the first few eigenvalues. Figure 2 shows 3 turning points, at $K_{0}=6.1, K_{1}=10.3$ and $K_{2}=14.2$, for $W=0.5$.

Figure 3 shows the shape of the $n=0$ mode, for various values of $W$. Since $h$ is a quantity determined by the solution process, it is inappropriate to plot $Y=y / h$ itself, as this does not demonstrate the variation in the absolute height of the sheet. An absolute measure of the sheet's height in any mode is

$$
\bar{y}=y D /\left(\rho U^{2} L^{4}\right)=Y K_{n}^{-4},
$$




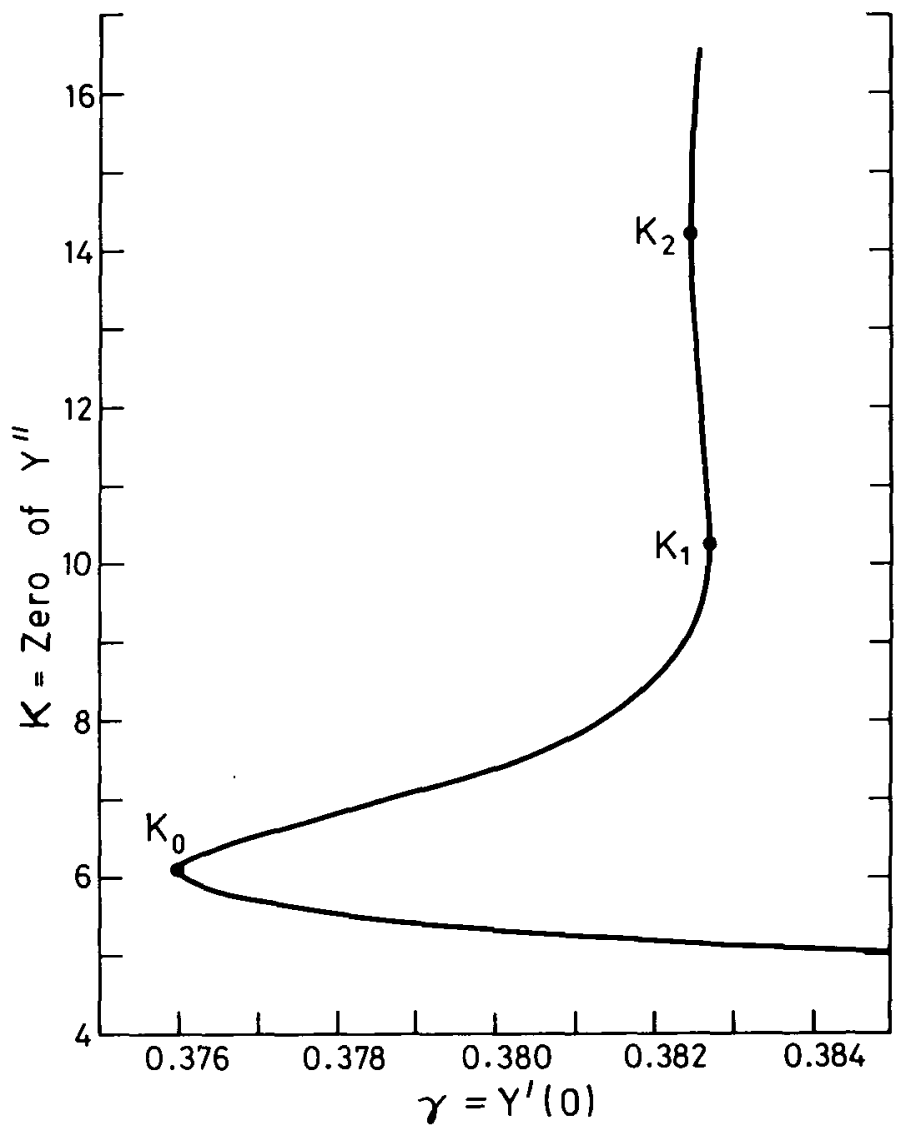

Figure 2. Example of graphical solution of boundary-value problem. Plot of zero of $Y^{\prime \prime}(X)$ (that is, a computed value of $X=K$ such that $Y^{\prime \prime}(K)=0$ ), against $\gamma=Y^{\prime}(0)$, at $W=0.5$. Turning points where $d \gamma / d K=0$ yield the required eigenvalues $K=K_{n}, 3$ of which are shown here.

and this is plotted against $x / L$ in Figure 3, for $n=0$. The horizontal line at $W=0$ corresponds to the linearized solution with $w \equiv 0$, that is, has $Y \equiv 1$, $\bar{y}=K_{0}^{-4}=0.00200$. For $W=0.1$, the linearized solution (4.7) predicts the shape of the sheet quite accurately, the main non-linear effect being an increase in the eigenvalue $K_{0}$ from 4.73 to 4.90 , and hence a corresponding reduction in the trailing-edge height $h$.

As $W$ increases (that is, the sheet becomes heavier) it must fly closer to the ground in order to provide enough aerodynamic force to support its weight, and, in the limit as $W \rightarrow 1$, the sheet lies flat on the ground. As we have seen, no solution is possible for $W>1$. At intermediate values of $W$, bending occurs, and, for $n=0$, such bending is symmetric about mid-chord. 


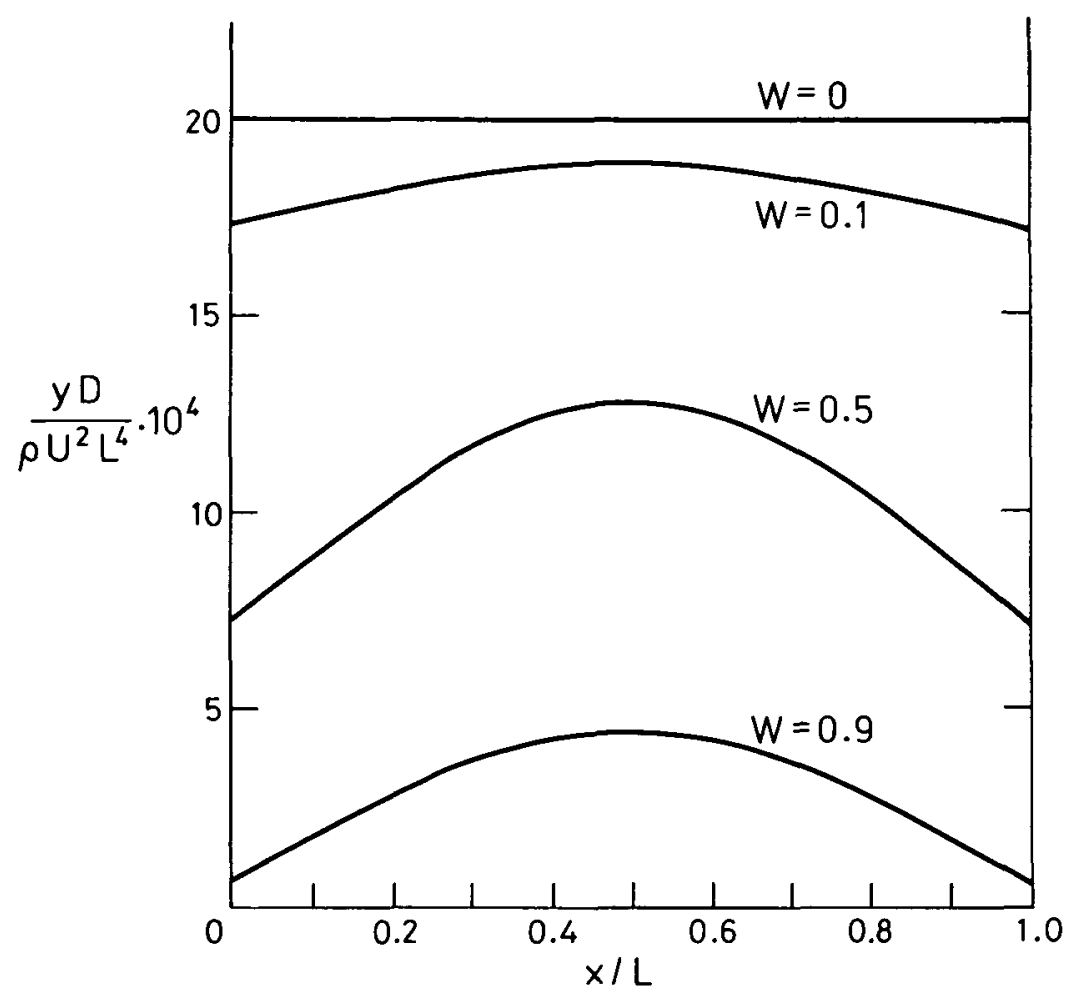

Figure 3. Sheet configuration in the first symmetric mode $n=0$, for various values of the weight parameter $W$.

Figure 4 shows corresponding results for the first odd mode $n=1$. This mode is almost antisymmetric about the mid-chord point. The sheet possesses a non-zero average angle of attack, and this mode can be thought of as a distortion of a rigid plate at an angle of attack. The main component of this distortion is an increased clearance in the aft half, that increases the local lift there, and hence shifts the centre of pressure back to mid-chord.

Note the factor of 10 difference in the scale of the vertical height between Figures 3 and 4 . The general size of the clearance is reduced in going from mode 0 to mode 1 by a factor of 8 or more for all values of $W$, and, when $W$ is close to 1 , the clearance is very small indeed, for all modes higher than $n=0$. Thus, attention can be confined to the $n=0$ mode in practice, as this is the only likely low-drag configuration.

For any given mode, at fixed speed $U$ and weight $w$, the height of the sheet is inversely proportional to the flexural rigidity $D$. That is, an almost-rigid sheet must fly at a very low height. As we have noted, a completely rigid sheet could 


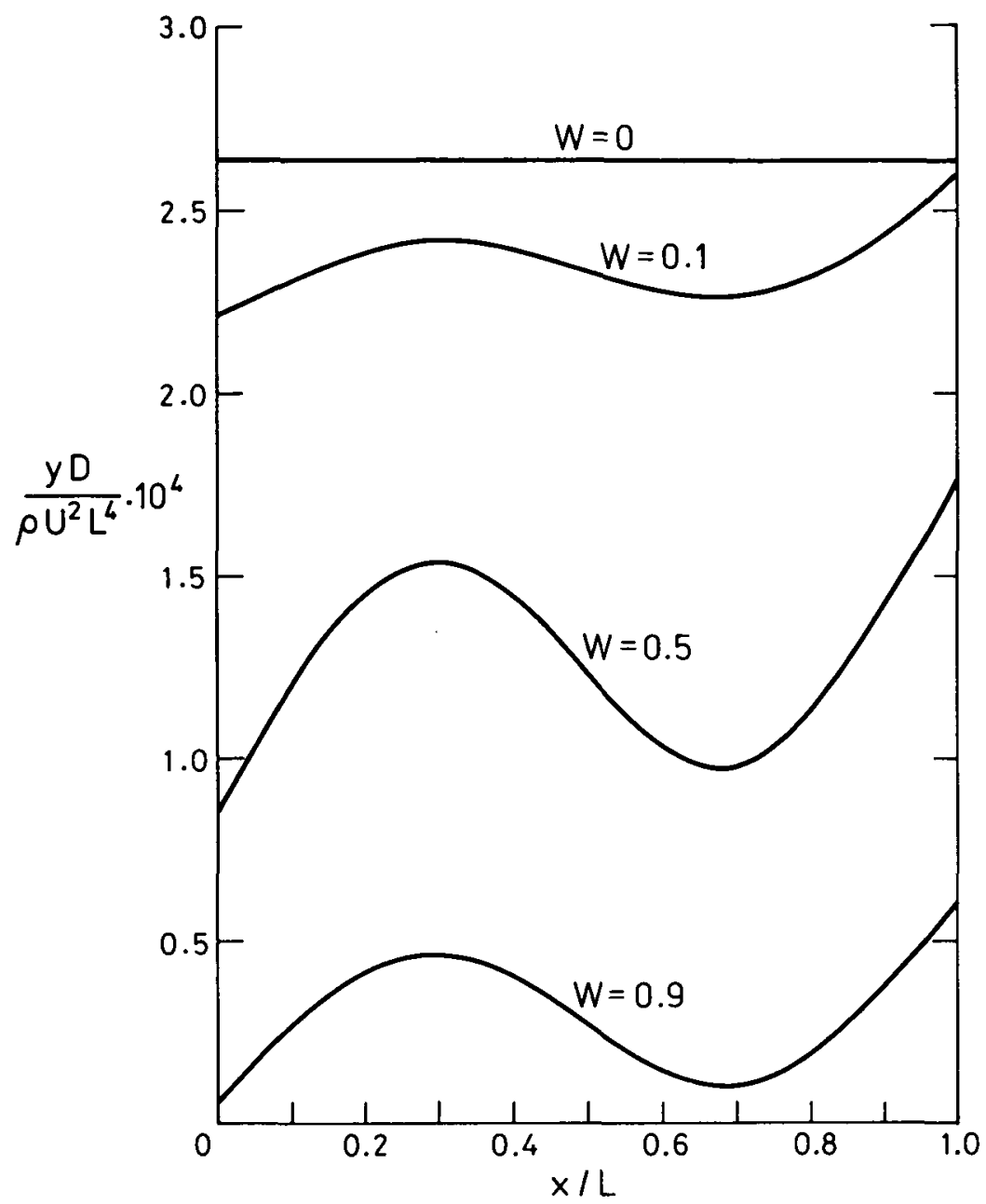

Figure 4. Sheet configuration in the first nearly-anti-symmetric mode $n=1$, for various values of the weight parameter $W$. Note factor of 10 vertical scale reduction from Figure 3.

only be supported by a uniform stagnation pressure obtained by causing its trailing edge height to vanish.

At the other extreme, the apparent conclusion is that, as the rigidity tends to zero, the height tends to infinity. Although this result only applies over a limited range such that the clearance/chord ratio remains small, it does suggest that highly flexible sheets should be capable of sliding further, their greater flying height reducing the drag. In practice, however, sheets that are too flexible do not slide well because they are hard to get started. 


\section{Conclusion}

The results presented here demonstrate the theoretical feasibility of purely aerodynamic support of sufficiently light flexible uniform sheets, in steady motion very close to a plane surface. The condition $w<\frac{1}{2} \rho U^{2}$ quantifies the degree of lightness. The main effect of the flexibility $D$ is to determine the overall height at which the sheet flies. A stiffer sheet must gain its necessary lift by flying closer to the ground, while bending less.

Nothing in the present work should be interpreted as an assertion that heavy or non-flexible sheets cannot slide at all. Sheets with $w>\frac{1}{2} \rho U^{2}$ cannot derive sufficient lift aerodynamically for steady motion. There are then two possibilities for apparent easy sliding. Firstly, the motion could be unsteady, but long-lasting, for a range of values of $U$ not too much below $(2 w / \rho)^{1 / 2}$. The sheet then decelerates and descends slowly, while flying in an air-supported low-drag configuration. This unsteady explanation would also be needed to account for sliding of completely non-flexible sheets, even if $w<\frac{1}{2} \rho U^{2}$. In any case, the whole concept of steady sliding is at best an approximation, since drag forces are continually slowing the sheet down.

The second possibility is that of conventional viscous air lubrication. The gap flow would then be nearly of a fully-developed Couette nature, with a substantially linear profile, and a pressure that is determined by the small extent of non-uniformity of the gap, via Reynolds' equation [1]. In principle, such a bearing-type pressure has no limit at all, so that arbitrarily-heavy sheets can be supported. However, for free sliding, the drag in such a mode is bound to be much higher than that for pure aerodynamic support.

It should be noted that the same requirement for flexibility occurs for viscous support, in that the centre of pressure is not in general located at mid-chord, for a bearing surface that is flat. In contrast to the aerodynamic case, however, the pressure distribution is biased toward the narrowest part of the gap, that is, toward the rear half of a rigid sheet at positive angle of attack. Again, the conclusion must be that a non-flexible free sheet cannot slide steadily in pure lubrication flow. The opposite nature of the aerodynamic and viscous asymmetries does suggest, however, that in a suitable interpolating regime, the centre of pressure may lie at mid-chord, and work is continuing on this extension of the present analysis.

Another desirable extension of the present work would be to study the stability of the equilibrium configurations determined here. That is, all that has been demonstrated here is that certain modes of steady motion do exist. These modes could be unstable. Indeed, it is clear that attempts to propel very thin sheets are often thwarted by an instability in which the leading edge rises rapidly. The 
possibility therefore exists that stability is possible only for sufficiently stiff sheets.

\section{Acknowledgement}

All computations presented here were performed on a TRS-80 microcomputer. Useful discussions are acknowledged with Jean-Marc Vanden Broeck and Len Schwartz, and the comments of the referees are appreciated.

\section{References}

[1] A. Cameron, Principles of lubrication (Longmans, London, 1966).

[2] L. H. Donnell, Beams, plates and shells (McGraw-Hill, New York, 1976).

[3] T. Strand, W. W. Royce and T. Fujita, "Cruise performance of channel-flow ground-effect machines", J. Aero. Sci. 29 (1962), 702-711.

[4] Sir Geoffrey Taylor, Low-Reynolds-number flows (16mm color sound film, Encyclopaedia Britannica Educational Corporation, Chicago, Illinois, 1967).

[5] E. O. Tuck, "A non-linear unsteady one-dimensional theory for wings in extreme ground effect", J. Fluid Mech. 98 (1980), 33-47.

[6] E. O. Tuck, "Steady flow and static stability of airfoils in extreme ground effect", J. Eng. Math. 15 (1981), 89-102.

[7] S. E. Widnall and T. M. Barrows, "An analytic solution for two- and three-dimensional wings in ground effect", J. Fluid Mech. 41 (1970), 769-792.

Department of Applied Mathematics

University of Adelaide

GPO Box 498

Adelaide

South Australia 5001 